
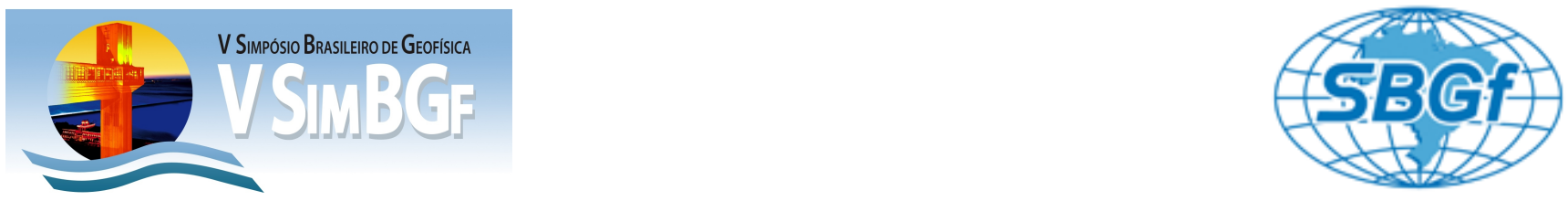

\title{
Sísmica de reflexão rasa: análise de velocidades de ondas convertidas PS
}

\author{
Éderson Ribeiro da Silva e Liliana Alcazar Diogo
}

Instituto de Astronomia, Geofísica e Ciências Atmosféricas - Universidade de São Paulo (IAG-USP)

Copyright 2012, SBGf - Sociedade Brasileira de Geofísica

Este texto foi preparado para a apresentação no V Simpósio Brasileiro de Geofísica, Salvador, 27 a 29 de novembro de 2012. Seu conteúdo foi revisado pelo Comitê Técnico do V SimBGf, mas não necessariamente representa a opinião da SBGf ou de seus associados. É proibida a reprodução total ou parcial deste material para propósitos comerciais sem prévia autorização da SBGf.

\section{Resumo}

Neste trabalho estudamos o problema de análise velocidades de ondas convertidas PS na escala de investigação rasa. Várias equações tem sido propostas com a finalidade de obter uma melhor aproximação para os tempos dos eventos de reflexão não hiperbólicos. $\mathrm{Na}$ primeira fase do estudo foram gerados dados sintéticos para avaliarmos a adequação das equações de tempo a diferentes modelos geológicos. Para as equações escolhidas desenvolvemos um procedimento de análise de velocidades cuja incógnita é apenas a velocidade da onda S.

De um modo geral, concluiu-se que é possível interpretar as ondas convertidas PS na escala de investigação rasa até um afastamento máximo igual a 2,5 vezes a profundidade do refletor, mas com limitações do número de camadas em subsuperfície.

\section{Introdução}

Ondas convertidas (PS) podem fornecer importantes informações sobre a velocidade das ondas de cisalhamento (S) e sobre os parâmetros do meio responsáveis pela propagação das ondas $\mathrm{P}$ e $\mathrm{S}$. Como relatado em Grechka \& Tsvankin (2002), pode-se reconstruir o tempo de percurso da reflexão SS através das reflexões PP e PS.

Para um grande número de cenários de exploração, ondas PS fornecem informações valiosas sobre estruturas em subsuperfície ou propriedades do meio complementando a interpretação inferida através dos dados das ondas PP convencionais.

Para as ondas convertidas PS, o moveout é intrinsecamente não hiperbólico, uma vez que cada metade do caminho é percorrida com uma velocidade diferente. Além disso, na escala de investigação rasa devido a sobreposição das ondas superficiais e de outros eventos coerentes é comum que as reflexões sejam observadas com nitidez apenas em afastamentos por vezes maiores do que a profundidade do refletor.

Neste trabalho avaliamos várias equações desenvolvidas para a aproximação dos tempos dos eventos de reflexão não hiperbólicos.

\section{Metodologia}

Qualquer equação de tempo que não seja a aproximação hiperbólica possui mais do que duas incógnitas. A maioria possui três incógnitas: o tempo normal (ou de afastamento nulo); a velocidade de Vrms do pacote acima do refletor e um fator de heterogeneidade que indica 0 quanto a variação dos tempos com 0 afastamento difere da aproximação hiperbólica.

Os métodos de análise de velocidades fundamentam-se no ajuste da equação de tempo de trânsito às reflexões identificadas nos registros sísmicos. Nesse trabalho avaliamos as principais fórmulas descritas na literatura propostas por diversos autores e reavaliadas por Blias (2007). A seguir descreveremos apenas as equações que apresentaram melhor ajuste aos dados exatos.

\section{Fórmula da hipérbole deslocada}

Com o intuito de melhorar a análise de velocidades em afastamentos longos, Malovichko (1978) criou a aproximação denominada de hipérbole deslocada, investigada também por Castle (1994). Essa equação também pode ser empregada em outras situações em que o comportamento de $\mathrm{t}(\mathrm{x})$ seja não hiperbólico, como é o caso das ondas convertidas PS, sendo dada por

$$
t_{p s}(x)=\left(1-\frac{1}{S}\right) t_{0 p s}+\frac{1}{S} \sqrt{t_{0 p s}^{2}+S \frac{x^{2}}{V_{p s}^{2}}}
$$

Onde: Vps é a velocidade de rms considerando as velocidades intervalares das ondas $\mathrm{P}$ e S; e a constante $S$ é chamada de coeficiente de heterogeneidade, dado por:

$$
S=\frac{\mu_{4}}{\mu_{2}^{2}} \quad \mu_{j}=\sum_{k=1}^{n} t_{k} V_{k}^{j} / \sum_{k=1}^{n} t_{k}
$$

\section{Fórmula de Stovas Alterada}

Após alguns testes percebemos que, alterando o valor da constante $c$ na fórmula a seguir, conseguimos deslocar 0 final da curva de tempo de trânsito de modo a aproximála aos valores exatos, $c=1$ corresponde a fórmula original utilizada por Stovas e Ursin (2007) para análise de ondas convertidas PS,

$$
t_{p s}(x)=\sqrt{t_{0 p s}^{2}+\frac{x^{2}}{V_{p s}^{2}}-\frac{(S-1) x^{4}}{4 V_{p s}^{4}\left(t_{0 p s}^{2}+\frac{(S-c)}{2} \frac{x^{2}}{V_{p s}^{2}}\right)}}
$$




\section{Resultados}

As figuras a seguir apresentam, para alguns dos modelos testados, a diferença entre os valores das curvas de tempo de trânsito usando diferentes aproximações e a curva exata obtida pelo método do traçado de raio.

Para avaliar a influência da razão $\mathrm{Vp} / \mathrm{Vs}$ no comportamento das curvas de tempo, utilizamos dois modelos com uma interface na mesma profundidade e diferentes valores da razão Vp/Vs (Figuras 1 e 2). Os outros dois exemplos visam avaliar a influência do número de camadas (Figuras 3 e 4, respectivamente para duas e três camadas). Para tal, a profundidade da interface alvo foi mantida a mesma, assim como a razão $\mathrm{Vp} / \mathrm{Vs}$ da última camada.

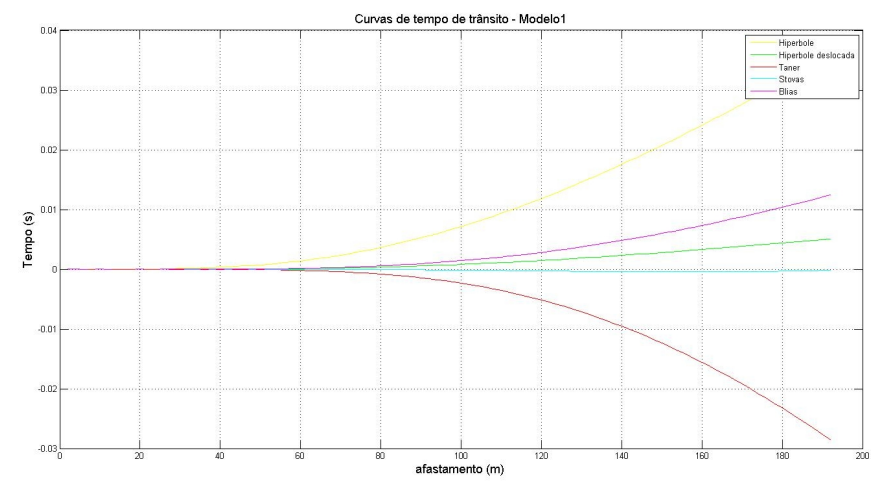

Figura 1: Modelo com 1 camada de profundidade 50m; $V p=1500 \mathrm{~m} / \mathrm{s}$ e $V s=452 \mathrm{~m} / \mathrm{s} \quad(\mathrm{z}=50 \mathrm{~m}$ e $\mathrm{Vp} / \mathrm{Vs}=3.3)$.

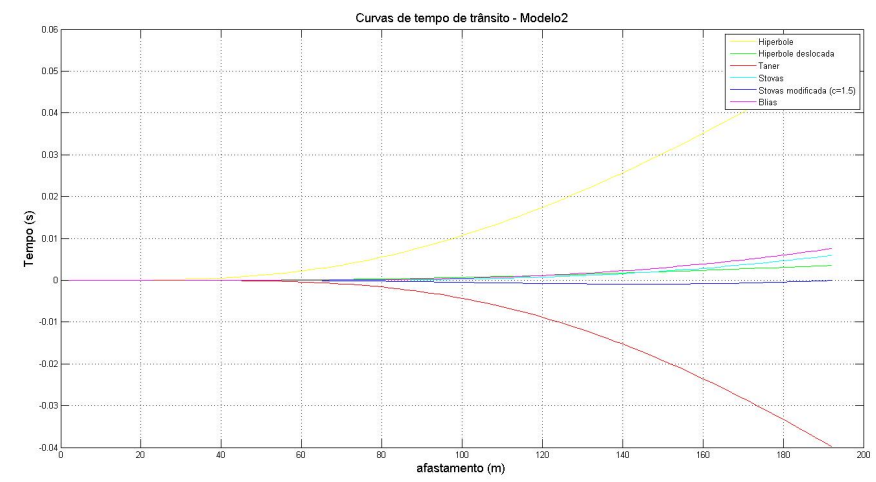

Figura 2: Modelo com 1 camada de profundidade $50 \mathrm{~m}$; $V p=1650 \mathrm{~m} / \mathrm{s}$ e Vs=300 m/s (z=50 m e Vp/Vs=5.5).

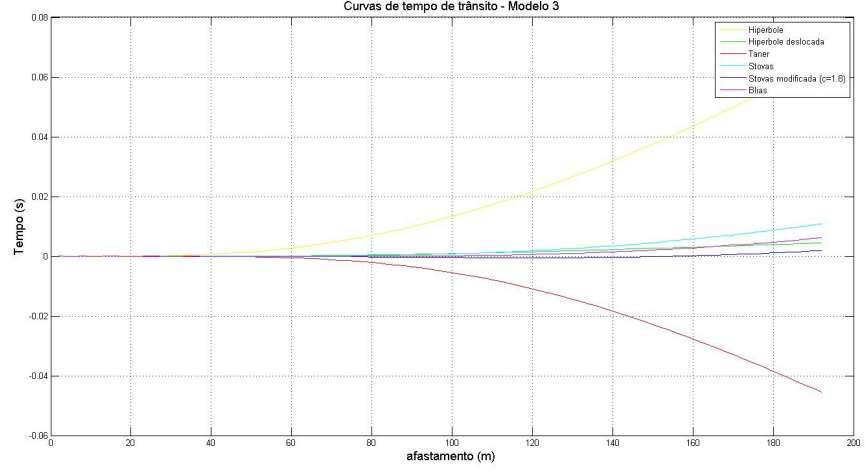

Figura 3: Modelo com 2 camadas de espessuras $5 \mathrm{~m}$ e $45 \mathrm{~m}$; $V p 1=370 \mathrm{~m} / \mathrm{s}, V s 1=112 \mathrm{~m} / \mathrm{s}, V p 2=1650 \mathrm{~m} / \mathrm{s}$ e Vs2 $=300 \mathrm{~m} / \mathrm{s}(\mathrm{z}=50$ m e $V p 1 / V s 1=3,3$ e $V p 2 / V s 2=5,5)$

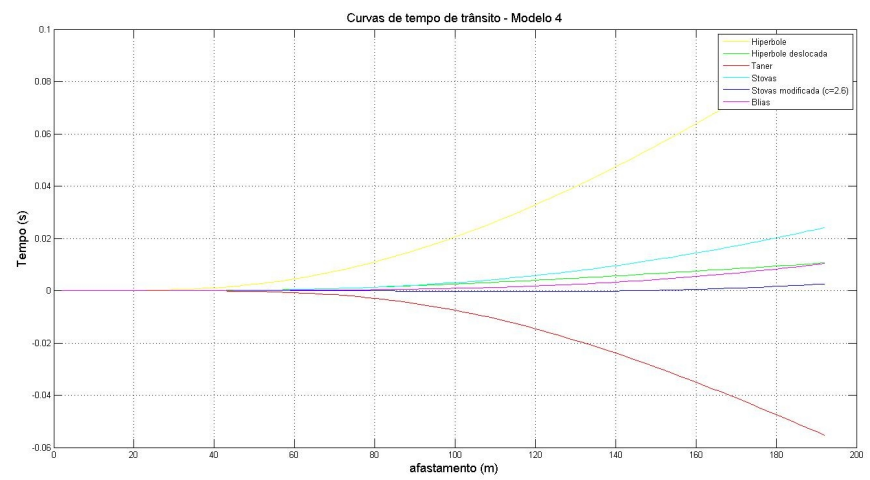

Figura 4: Modelo com 3 camadas de espessuras $5 \mathrm{~m}, 15 \mathrm{~m}$ e $30 \mathrm{~m} ; V p 1=370 \mathrm{~m} / \mathrm{s}, V s 1=112 \mathrm{~m} / \mathrm{s}, V p 2=600 \mathrm{~m} / \mathrm{s}, V s 2=200 \mathrm{~m} / \mathrm{s}$, $V p 3=1650 \mathrm{~m} / \mathrm{s}$ e $V \mathrm{~s} 3=300 \mathrm{~m} / \mathrm{s} \quad(\mathrm{z}=50 \quad \mathrm{~m}$ e $\quad \mathrm{Vp} 1 / \mathrm{Vs} 1=3,3$ $V p 1 / V s 2=3,0$ e $V p 3 / V s 3=5,5)$

\section{Análise de Velocidades}

Escolhidas as melhores equações para o cálculo dos tempos de trânsito, propomos realizar a análise de velocidades supondo a situação em que conhecemos a velocidade $\mathrm{Vp}$ e top, obtida da análise da onda $P$ refletida, e que extraímos o tempo normal (t0ps) com algum dos procedimentos convencionais baseados na equação da hipérbole. Desta forma, é possível calcular as curvas de tempo de trânsito variando apenas o valor da velocidade da onda $\mathrm{S}$ (Vs). As curvas, assim calculadas, serão plotadas sobre o sismograma e será escolhida visualmente a velocidade que gerou a curva que melhor se sobrepõe aos dados (Figuras 5 a 7 , respectivamente para os modelos 1 a 3). 


\section{Discussão e Conclusões}

De um modo geral, até um afastamento máximo igual a 2,5 vezes a profundidade do refletor, as equações de Stovas e hipérbole deslocada apresentam um bom ajuste aos valores exatos.

Para a razão afastamento/profundidade maior do que 2,5, o erro ao utilizar a equação de Stovas é mais significativo, conforme a razão $\mathrm{Vp} / \mathrm{Vs}$ do meio aumenta. Esse erro pode ser corrigido variando a constante (c) na equação de Stovas Alterada, entretanto como não encontramos uma relação da constante c com os parâmetros do meio, a equação de Stovas alterada não foi utilizada na análise de velocidades.

Para modelos geológicos com mais de duas camadas o método torna-se mais complicado, visto que precisaríamos conhecer as velocidades das ondas $\mathrm{P}$ e $\mathrm{S}$ das camadas superiores, tendo como incógnita apenas a velocidade $\mathrm{S}$ da última camada. Como essa situação pode ser difícil de se resolver na prática, a utilização do método proposto pode estar restrita a estruturas com apenas duas camadas, o que entretanto é bastante comum em situações geológicas rasas.

\section{Referências}

BLIAS, E.; Long-spreadlength approximations to NMO function for a multi-layered subsurface. CSEG Recorder, p. 36-42, March 2007.

CASTLE, R. J. A theory of normal moveout. Geophysics, v.59, n.06, p.983-999, 1994.

GRECHKA, V., TSVANKIN, I., PP + PS = SS: Geophysics, v. 67, 1961-1971, 2002.

MALOVICHKO, A. A. A new representation of the traveltime curve of reflected waves in horizontally layered media. Applied Geophysics, n.91, p.47-53, 1978 (in Russian).

STOVAS, A.; URSIN, B. Estimation of layer parameters for linear P- and S-wave velocity functions. Geophysics, v. 72, p. U27-U30, 2007.

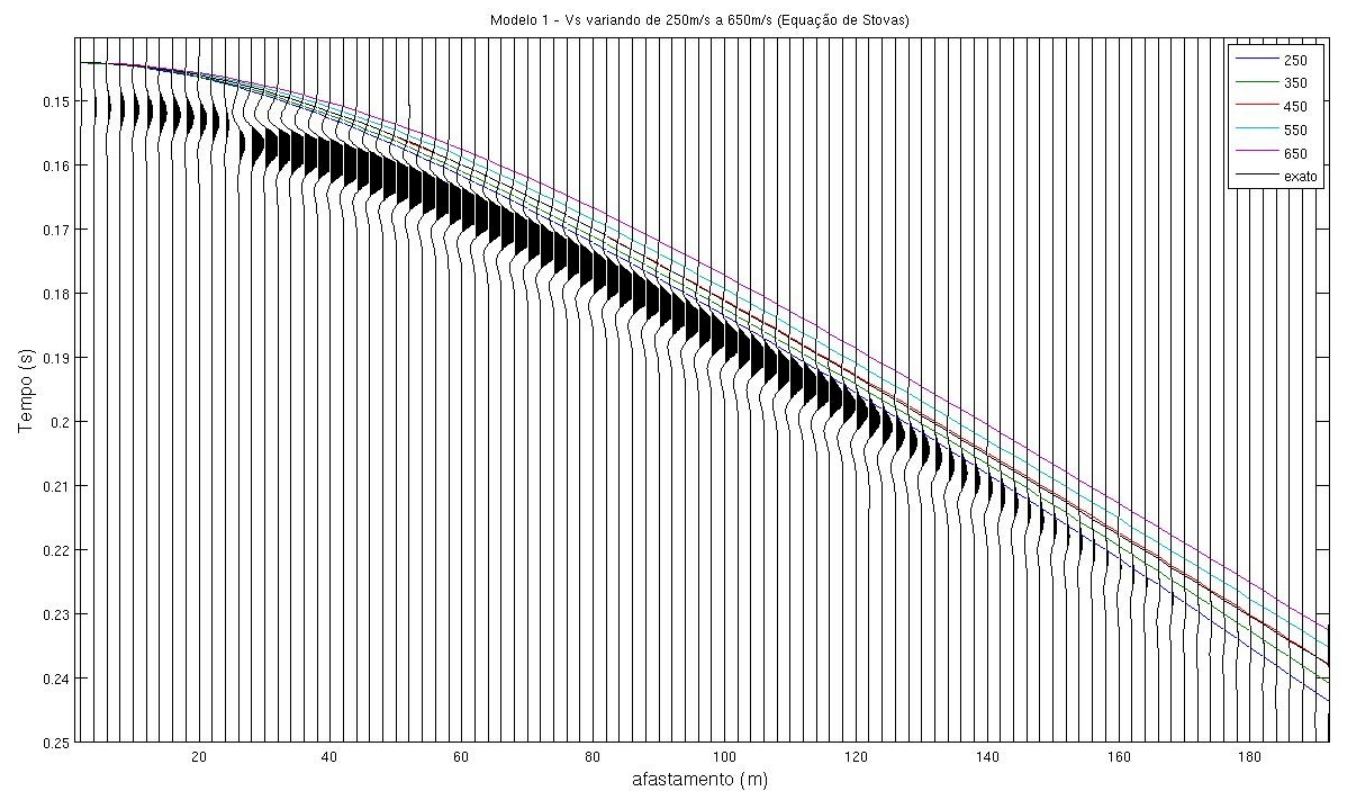

Figura 5: Curvas de tempo usando a equação de Stovas para diferentes velocidades da onda Sobrepostas aos dados sísmicos do modelo 1 (Figura 1). Valor correto igual a $452 \mathrm{~m} / \mathrm{s}$. 


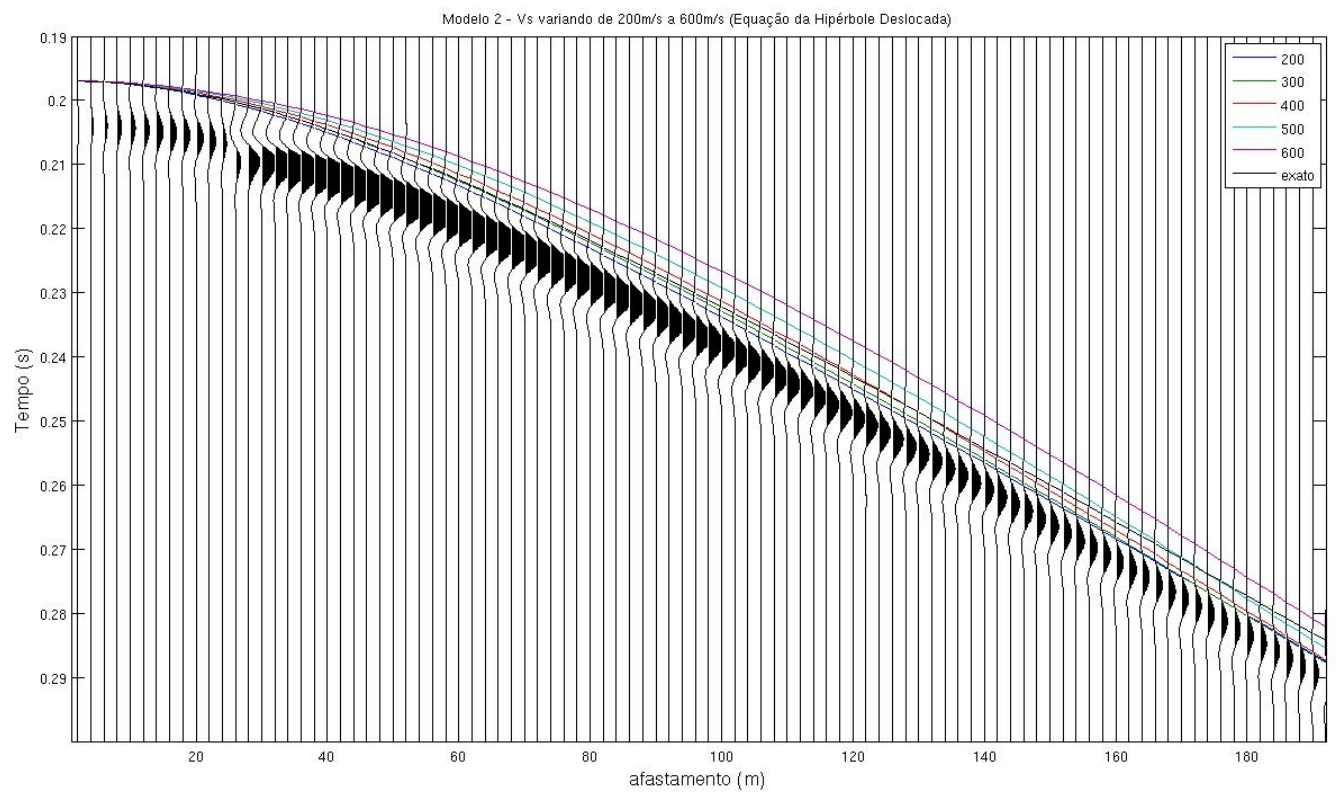

Figura 6:Curvas de tempo usando a equação da hipérbole deslocada para diferentes velocidades da onda $S$ sobrepostas aos dados sísmicos do modelo 2. (Figura 2). Valor correto igual a $300 \mathrm{~m} / \mathrm{s}$.

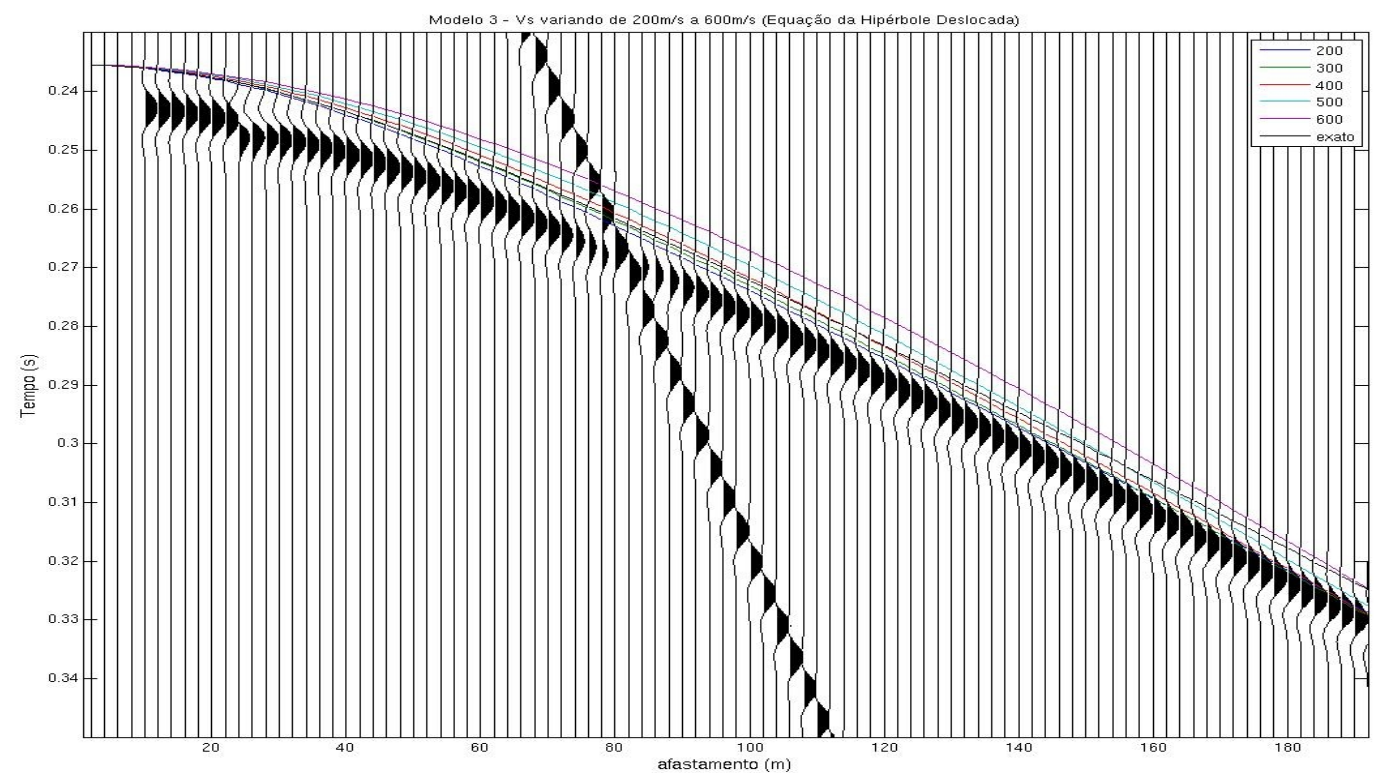

Figura 7: Curvas de tempo usando a equação da hipérbole deslocada para diferentes velocidades da onda S sobrepostas aos dados sísmicos do modelo 3. (Figura 3). Valor correto igual a $300 \mathrm{~m} / \mathrm{s}$. 